\section{Haemodynamic challenge in non-restrictive atrial septal defect}

\section{CLINICAL INTRODUCTION}

A 55-year-old hypertensive man presented with history of New York Heart Association class II exertional dyspnoea for 3 years. The arterial pulse was normal. His blood pressure was $180 / 100 \mathrm{~mm} \mathrm{Hg}$. Jugular venous waveforms revealed prominent 'a waves' at the root of neck though the mean pressure appeared to be normal. A wide fixed split second heart sound and midsystolic murmur at the pulmonary area were noted. A soft third heart sound and flow murmur at the left lower sternal border suggested significant pre-tricuspid left-to-right shunt.

Echocardiography showed a $32 \mathrm{~mm}$ ostium secundum atrial septal defect (ASD) with deficient rims. Volume overload of the right atrium and right ventricle was noted. Left ventricular (LV) systolic function was normal with no regional wall motion abnormalities. Pulmonary venous return was normal. All cardiac valves were normal and competent. He was taken up for cardiac catheterisation prior to ASD surgical closure.

Room air oximetry suggested a left-to-right shunt of 2.2:1. The haemodynamic tracings are given in figure 1 . Coronary angiogram revealed right dominant normal coronaries.

\section{QUESTION}

What is the haemodynamic issue that affects the current management plan and what should be the management strategy?

a. Severe pulmonary hypertension. Patient should be initiated on pulmonary vasodilators and a follow-up cardiac catheterisation should be done.

b. Constrictive pericarditis. Pericardiectomy must be considered.

c. Biventricular restrictive cardiomyopathy. ASD closure must be deferred.

d. LV diastolic dysfunction. Patient should be initiated on a diuretic prior to ASD closure.

e. Significant subvalvar pulmonary stenosis. Concomitant infundibular resection needs to be planned.

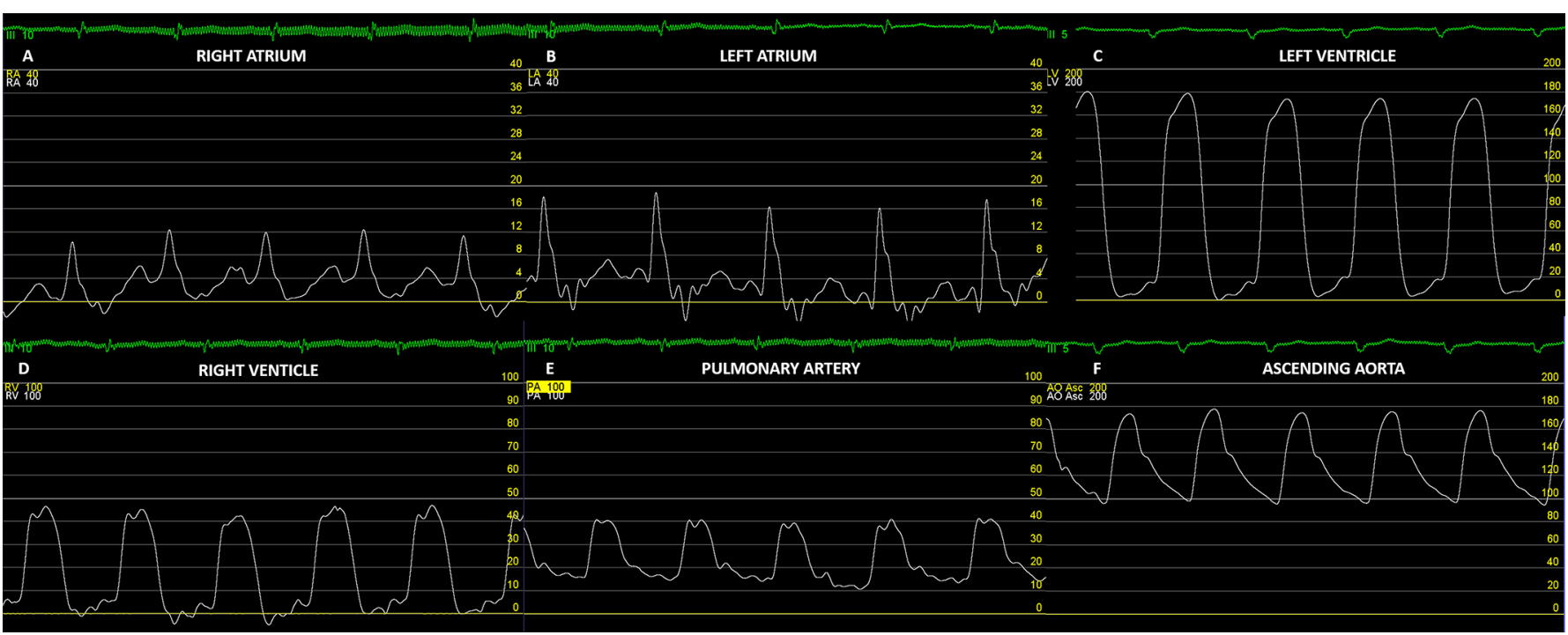

Figure 1 The hemodynamic tracings presented are that of the right atrium (Panel A), left atrium (Panel B), left ventricle (Panel C), right ventricle (Panel D), main pulmonary artery (Panel E) and ascending aorta (Panel F). 


\section{CORRECT ANSWER: D.}

The haemodynamic tracings demonstrate LV diastolic dysfunction with elevated LV end-diastolic pressure and consequent diastolic heart failure. Non-restrictive ASD is classically associated with $\mathrm{v}$ wave $\geq$ a wave phenomenon in the young while older adults often show marginally larger 'a wave' in right atrial pressure tracings. In impaired LV relaxation, vigorous left atrial contraction ensures that the left atrial mean pressure is normal even when LV end-diastolic pressures are elevated. The resultant prominent left atrial 'a wave' is easily reflected in the jugular venous waveforms in the presence of a large ASD. Careful evaluation of jugular venous waveforms can thus help clinically identify LV diastolic dysfunction and take measures to avert pulmonary oedema during ASD closure by optimal diuresis, consideration of fenestrated patch ASD closure and careful postprocedure monitoring. The patient underwent ASD closure with a fenestrated Goretex patch after preoperative diuresis for 2 weeks. He was discharged on the sixth postoperative day and is doing well on follow-up.

Pulmonary hypertension is moderate, and with a shunt fraction of $>2: 1$, the patient is clearly a candidate for ASD closure. Despite the elevated 'a waves' in either atria, the mean atrial pressures are not elevated and the right ventricular end-diastolic pressure is normal, which rules out biventricular restrictive cardiomyopathy or constrictive pericarditis in the presence of an ASD. Constrictive haemodynamics is unlikely in the presence of normal dip-diastolic pressures of the ventricles that suggests normal early diastolic ventricular filling. Right ventricular outflow tract obstruction, another cause of elevated 'a wave' in the atrial tracings, is ruled out by the absence of systolic pressure gradients between the right ventricle and pulmonary artery.

\section{Arun Gopalakrishnan, Krishna Kumar Mohanan Nair, Sanjay Ganapathi}

Department of Cardiology, Sree Chitra Tirunal Institute for Medical Sciences and Technology, Thiruvananthapuram, Kerala, India

Correspondence to Dr Sanjay Ganapathi, Department of Cardiology, Sree Chitra Tirunal Institute for Medical Sciences and Technology, Thiruvananthapuram, Kerala 695011, India; drsanjayganesh@gmail.com

Contributors All authors prepared the manuscript and approved the final version. Competing interests None declared.

Patient consent Obtained.

Ethics approval The study was conducted in accordance with the ethical standards laid down in the 1964 Declaration of Helsinki and its amendments.

Provenance and peer review Not commissioned; externally peer reviewed.

(c) Article author(s) (or their employer(s) unless otherwise stated in the text of the article) 2018. All rights reserved. No commercial use is permitted unless otherwise expressly granted.

\section{Check for updates}

To cite Gopalakrishnan A, Mohanan Nair KK, Ganapathi S. Heart Asia 2018;10:e011014.

Heart Asia 2018;10:e011014. doi:10.1136/heartasia-2018-011014

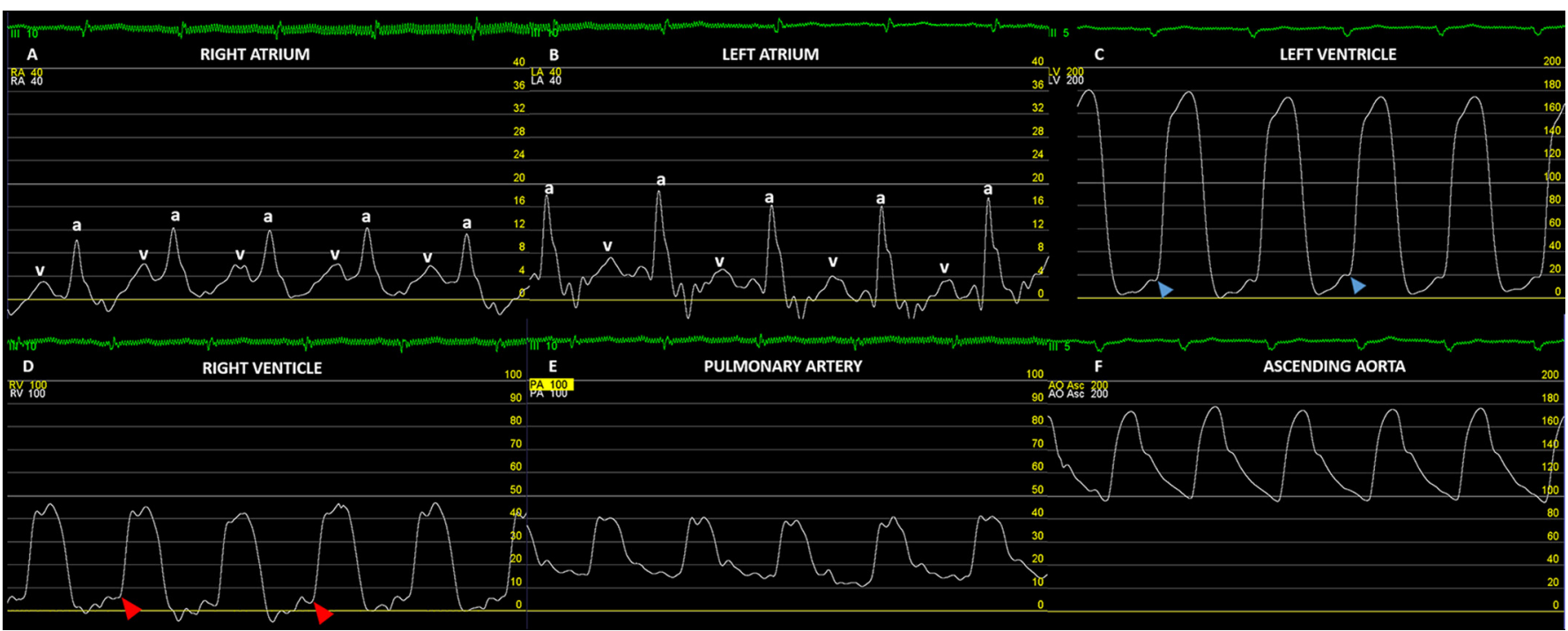

Figure 2 The haemodynamic tracings presented are that of (Panel A) right atrium (RA), (Panel B) left atrium (LA), (Panel C) left ventricle (LV), (Panel D) right ventricle (RV), (Panel E) main pulmonary artery (PA) and (Panel F) ascending aorta (AO). Blue arrowhead points to the elevated LV end-diastolic pressure (18-20 mm Hg). Red arrowhead points to the normal RV end-diastolic pressure (5 mm Hg). Prominent elevated 'a waves' are notable in the left and right atrial pressure tracings. 